Int. J. Dev. Biol. 50: 455-461 (2006)

doi: $10.1387 / \mathrm{ijdb} .052073 \mathrm{mt}$

Original Article

\title{
Dynamic distribution of the replacement histone variant H3.3 in the mouse oocyte and preimplantation embryos
}

\author{
MARIA-ELENA TORRES-PADILLA, ANDREW J. BANNISTER ${ }^{1}$, PAUL J. HURD ${ }^{1}$, TONY KOUZARIDES ${ }^{1}$ and \\ MAGDALENA ZERNICKA-GOETZ*
}

The Wellcome Trust/Cancer Research UK Gurdon Institute, and ${ }^{1}$ Department of Pathology, University of Cambridge, U.K.

\begin{abstract}
Upon fertilization, the gametes undergo a drastic reprogramming that includes changes in DNA methylation and histone modifications. Currently, it is not known whether replacement of the major histones by histone variants is also involved in these processes. Here we have examined the expression and localization of the histone variant $\mathrm{H} 3.3$ in early mouse embryogenesis. We show that $\mathrm{H3.3}$ is present in the oocyte as a maternal factor. It is then incorporated preferentially into the male pronucleus before genome activation, pointing towards an asymmetry in histone composition between the two pronuclei. This is in line with the male pronucleus bearing transcriptional activation first. The same distribution was observed when we followed the localisation of a tagged version of H3.3. We detected H3.3 in the nuclei of mouse embryos in all of the stages analysed, from the zygote to the blastocyst stage, suggesting that the epigenetic mechanisms in the early embryo not only involve changes in histone modifications but may also include histone replacement.
\end{abstract}

KEY WORDS: histone variant, early mouse embryo, reprogramming, replacement histone

\section{Introduction}

The removal and acquisition of new epigenetic marks during preimplantation development is essential to ensure the totipotency required for sustaining further development. In the zygote, changes in the epigenetic status of the parental genomes may also be needed for the activation of zygotic transcription. Covalent modifications of histones and DNA methylation are considered as the most important epigenetic marks in preimplantation development (Li, 2002, Morgan et al., 2005).

In the early zygote, histones $\mathrm{H} 3$ and $\mathrm{H} 4$ are synthesized from maternal mRNA, whereas the synthesis of histones $\mathrm{H} 2 \mathrm{~A}, \mathrm{H} 2 \mathrm{~B}$ and $\mathrm{H} 1$, is delayed until the end of the first cell cycle (Wiekowski et al., 1997). Covalent modifications of histones contribute to the formation and maintenance of transcriptionally active or inactive chromatin domains. Acetylation of lysine $(\mathrm{K})$ residues is generally associated with transcriptional activation whereas methylation of lysine residues can have opposing outcomes (repression or activation) depending on the precise methylation site. Apart from the major core histones, whose synthesis is tightly linked to the Sphase of the cell cycle, some histones are synthesized from orphan genes throughout the cell cycle and are incorporated into the chromatin independently of replication (Kamakaka and Biggins, 2005). These 'replacement' histones are also covalently modified and can also be used to 'mark' the chromatin and hence regulate gene expression. Moreover, they can elicit different chromatin conformations upon assembly into the nucleosomes (Gautier et al., 2004). One interesting variant is H3.3, which can replace histone H3. The replication-independent deposition of histone variants allows the turnover of modification marks and hence a change of the heritable chromatin states. For example, upon gene activation, H3 carrying the repressive methyl H3K9 mark becomes replaced by H3.3 unmethylated at K9. Moreover, preferential deposition of $\mathrm{H} 3.3$ in transcriptionally active regions would result in perpetuation of epigenetic memory (Ahmad and Henikoff, $2002 b$, Schwartz and Ahmad, 2005). Although a growing number of reports support a role for the histone variants in regulating chromatin activity, their specific functions in vivo are not fully understood.

Several recent studies have analysed histone modifications in early embryos (Adenot et al., 1997, Arney et al., 2002, Lepikhov and Walter, 2004, Santos et al., 2005, Sarmento et al., 2004). However, to obtain a better understanding of the reprogramming taking place in the mammalian embryo, it is also necessary to define the components of the chromatin present throughout

Abbreviations used in this paper: GV, germinal vesicle; HA tag, hemagglutin tag; $\mathrm{PN}$, pronuclear stage; $\mathrm{h}$ phCG, hours after hCG injection.

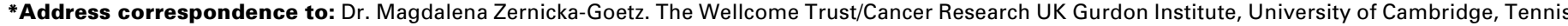
Court Road, Cambridge CB2 1QR, U.K. Fax+44-1223-634089. e-mail: mzg@mole.bio.cam.ac.uk
} 
preimplantation development. Here, we have characterized the localization of the replacement histone variant $\mathrm{H} 3.3$ in mouse oocytes and early embryos. The histone variant $\mathrm{H} 3.3$ was present in all the stages examined. In the zygote, it showed an asymmetric distribution between the male and female pronucleus. We also observed that the pattern of localization of $\mathrm{H} 3.3$ changes from a punctate to a more disperse nuclear staining after the 2-cell stage.

\section{Results}

Here, we were interested in examining the distribution of the replacement histone variant $\mathrm{H} 3.3$ in oocytes and preimplantation mouse embryos. The characterisation of the H3.3 antibody used in this study is shown in Figure 1A. Only the specific H3.3 peptide competes the antibody. Importantly, the H3.3 antibody does not recognise a recombinant purified $\mathrm{H} 3.2$ variant, testifying for the specificity of the antibody. Using this $\mathrm{H} 3.3$ specific antibody we analysed the distribution of H3.3 in the oocyte. The H3f3agene, encoding H3.3, was previously reported to be expressed in the growing oocyte (Couldrey et al., 1999). H3.3 clearly accumulates in the germinal vesicle (GV), but it is also detectable in the cytoplasm (Fig. 1B, left panel). After fertilisation, the maternal genome resumes meiosis from metaphase II and extrudes the second polar body containing the second set of haploid chromosomes. During this time, which corresponds to the pronuclear stage PNO (Adenot et al., 1997), H3.3 is only weakly dispersed in the cytoplasm with no obvious localisation in either the maternal or the paternal pronuclei (Fig. 1B, right panel).

The pronuclei are subsequently subject to cycles of decondensation and migrate towards the centre of the zygote. We first detect an enrichment of $\mathrm{H} 3.3$ in the pronuclei during decondensation around the PN2 stage. This enrichment is asymmetric between the two pronuclei: it is evident in the male pronucleus, but not in the female one. At this stage, a weak staining of the cytoplasm suggests localisation of H3.3 in the cytoplasm, probably reflecting translation, storage or association with chaperone molecules (Fig. 2A). Thus, the maternal genome

is associated with $\mathrm{H} 3.3$ in the $\mathrm{GV}$, but $\mathrm{H} 3.3$ is then undetectable in the maternal chromatin during pronuclear formation and its incorporation starts earlier in the male pronucleus. In PN3-PN4 zygotes the pronuclei increase in size and locate close to each other in the centre of the cell. At this stage, the enrichment of H3.3 is still slightly brighter in the male pronucleus, but the female pronucleus also shows accumulation of $\mathrm{H} 3.3$ (the signal/size ratio of the male pronucleus compared to the female one was $1.8 \pm$ $0.17, n=7$, as quantified with the Volocity software, Improvision)(Fig. 2A). Before mitosis, the pronuclei become apposed (PN5), at this stage $\mathrm{H} 3.3$ is present in the two pronuclei and a difference in $\mathrm{H} 3.3$ accumulation between the male and the female pronucleus is no longer detected (Fig. 2A). At the 2-cell stage H3.3 is present in the two nuclei of the two blastomeres (Fig. 2A).

H3.3 can be deposited via a replication-independent pathway that is triggered upon transcriptional activation (Janicki et al., 2004, Schwartz and Ahmad, 2005). We therefore wished to know whether the time when we first saw deposition of $\mathrm{H} 3.3$ in the zygote corresponded to the time when the genome activation occurs. Thus, we followed the incorporation of BrUTP in the zygote by immunofluorescence. When examined at 24h phCG (approximately PN3-PN4 stages), we do not detect BrUTP in either pronucleus, indicating that the zygote is not yet transcriptionally active at this stage (Fig. 2B). However, when examined at 27h phCG (PN5), we detect BrUTP staining throughout the nucleoplasm with sites of brighter intensity around the nucleolarlike bodies in the two pronuclei (Fig. 2B). This is consistent with the observation that the zygote undergoes transcriptional activation between 26h and 29h phCG (Aoki et al., 1997, Bouniol et al., 1995). H3.3 exhibits a characteristic punctate staining throughout the nucleoplasm at these early stages and in the oocyte, which persists from the PN3 zygote stage up to the 2-cell stage (Fig. 2C). This pattern is different to the one exhibited by the BrUTP incorporated in the zygote (Fig. 2B). Thus, our results suggest that histone $\mathrm{H} 3.3$ accumulates in the male pronucleus before the onset of transcription activation.

We performed another series of experiments to confirm our

A

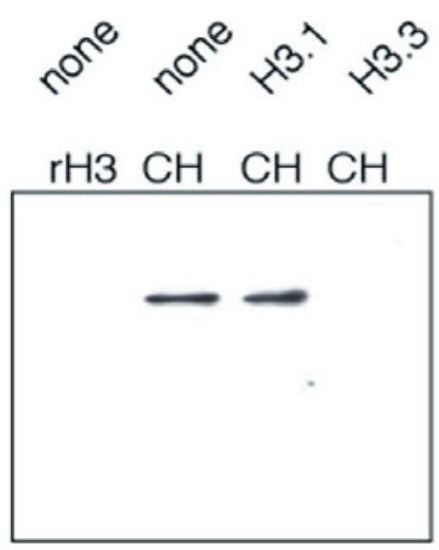

B

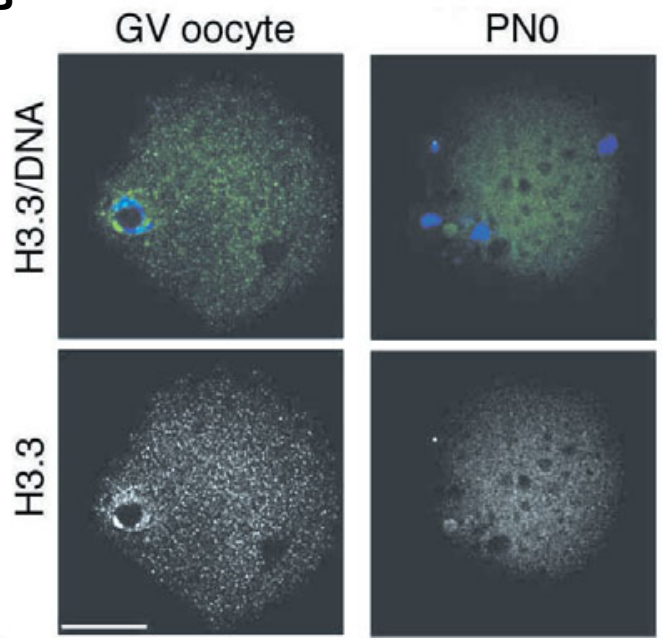

Fig. 1. Western blot confirming specificity of the H3.3 antibody (A) and immunochemistry revealing presence of Histone $\mathrm{H} 3.3$ in the germinal vesicle stage oocyte (B). (A) 2 micrograms of calf thymus core histones $(\mathrm{CH} ; 500 \mathrm{ng}$ each of histones $\mathrm{H} 2 \mathrm{~A}, \mathrm{H} 2 \mathrm{~B}, \mathrm{H} 3$ and $\mathrm{H} 4)$ and 1 microgram of bacterially purified and produced histone H3.2 (rH3) were resolved by SDS-PAGE, Western blotted to nitrocellulose and probed with the anti-H3.3 antibody. Antibody probing was performed in the absence or presence of competitor blocking peptide $(1 \mu \mathrm{g} / \mathrm{ml})$ as indicated above each lane on the panel. (B) Single confocal sections of a germinal vesicle stage (GV) oocyte and a zygote at the PNO stage (Adenot et al., 1997) stained with the H3.3 antibody (green) and TOTO-3 (DNA, blue). In the oocyte, H3.3

localises mainly to the germinal vesicle with some accumulation in the cytoplasm. After fertilization, before the extrusion of the second polar body during anaphase of the second meiotic division, H3.3 is dispersed in the cytoplasm. The head of the sperm (blue) is visible on the right hand side of the zygote. Merge images (top) and grayscale micrographs for H3.3 (bottom) are shown. Scale bar represents $40 \mu \mathrm{m}$. 
A

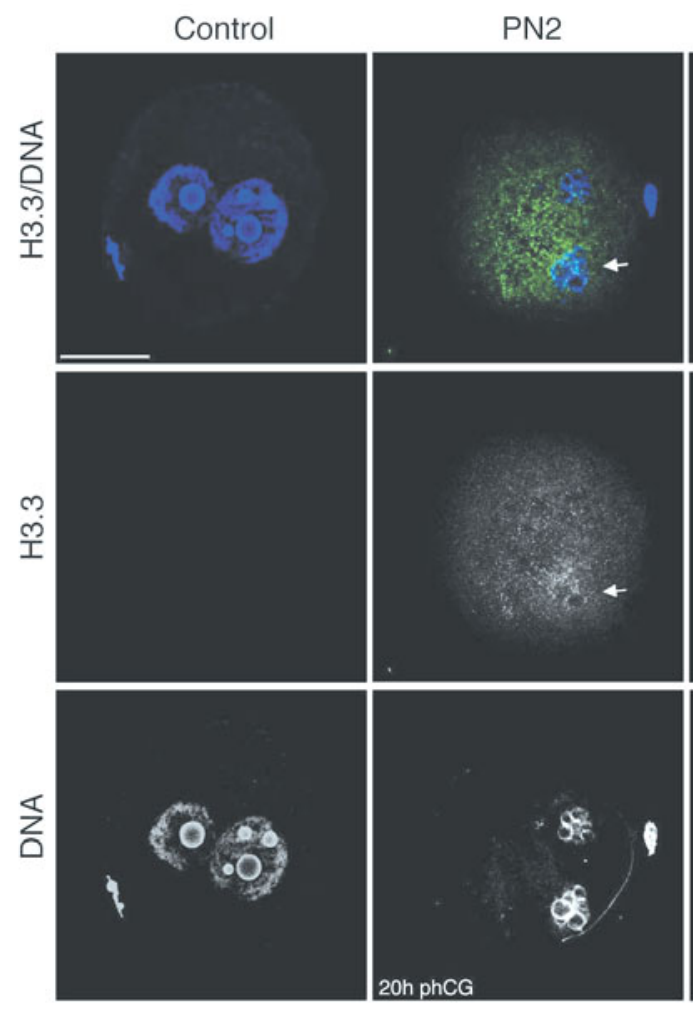

PN3-PN4
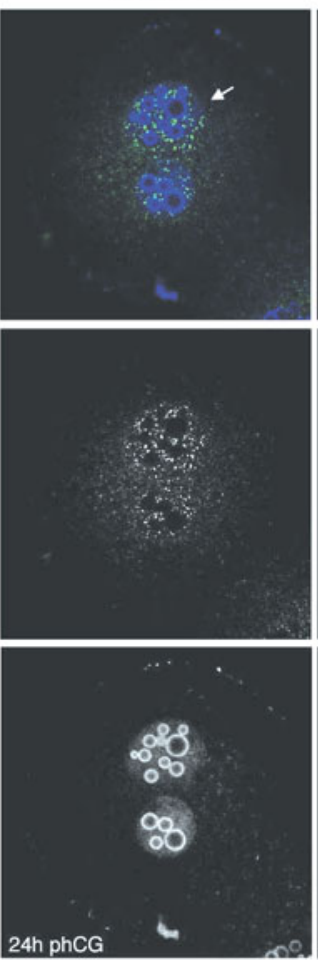

PN5
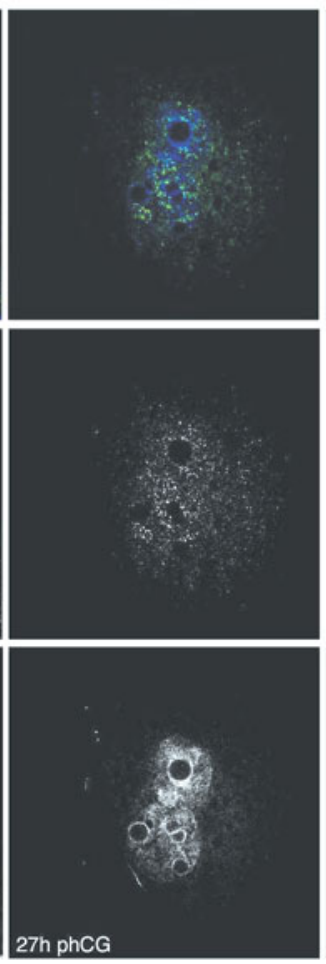

2-cell
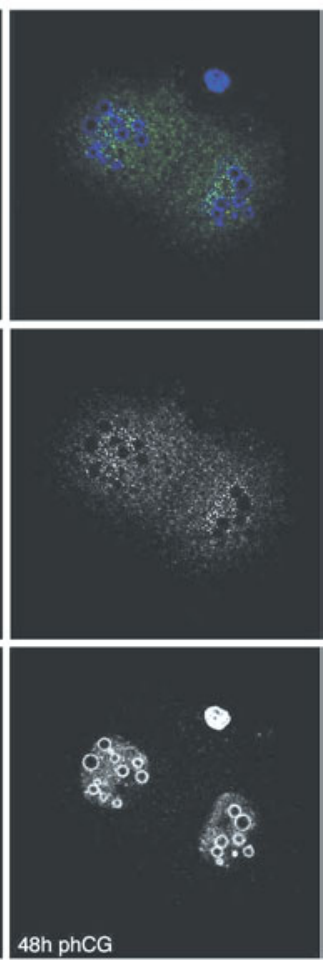

B

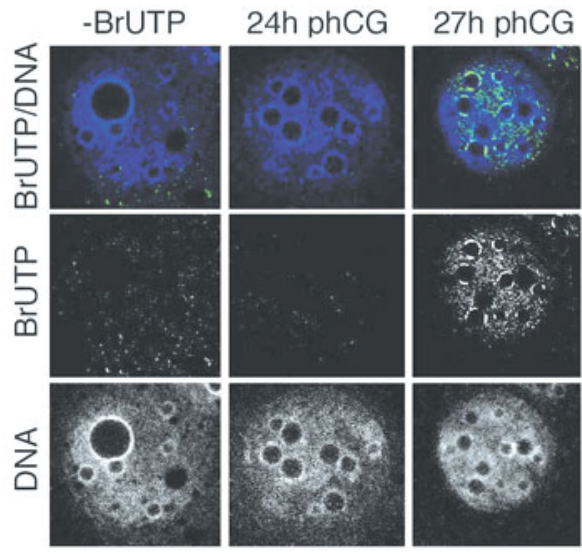

C

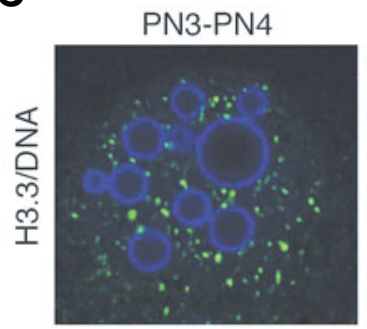

2-cell
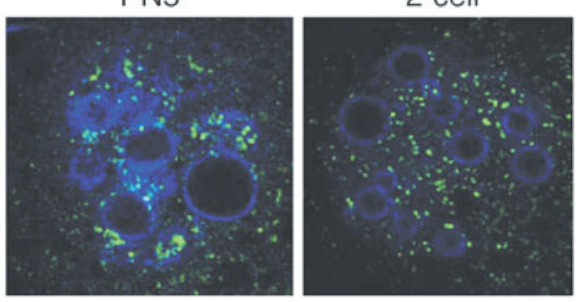

Fig. 2. Deposition of histone H3.3 in the zygote occurs first in the male pronucleus around the PN2 stage before the onset of transcription activation. (A) Embryos were collected at the indicated hours after hCG injection (h phCG) and processed for immunofluorescence for H3.3 (green). DNA is shown in blue in the merge panel (top). Classification of the pronuclear (PN) stages was done according to Adenot et al., (1997). For the control panel, a PN5 zygote for which only the secondary antibody was used is shown and the embryos were processed using the same confocal parameters. Shown are single confocal sections of representative embryos in which the maximum diameter of both pronucleiare on the same confocal plane. The male pronucleus is indicated with an arrow. Scale bar represents $40 \mu \mathrm{m}$. (B) Transcription activation is first detected in the zygote around the PN5 stage. Early zygotes were microinjected with BrUTP and cultured until the PN3-PN4 ( 24 h phCG) or the PN5 ( 27h phCG) stages, when they were fixed and processed for immunofluorescence using an anti-BrdU antibody (green). A non-injected embryo cultured until the PN5 stage is shown as negative control (-BrUTP). DNA is in blue in the top panel. Shown are single confocal micrographies of representative pronuclei at the indicated times after hCG injection. The pronucleus shown in all cases is the male and similar results were obtained for the female pronucleus. (C) Higher magnifications of representative pronucleiat the indicated zygote stages and of a 2-cell stage nucleus showing punctate localization of H3.3. Shown are merged single confocal sections where H3.3 is shown in green and DNA in blue.

results. We injected mRNA for $\mathrm{HA}$-tagged $\mathrm{H} 3.3$ into zygotes at the fertilisation cone stage, that is, right after fertilisation and before pronuclear formation. We then analysed the distribution of H3.3$\mathrm{HA}$ at similar pronuclear stages as above. The results shown in Figure $3 \mathrm{~A}$ demonstrate that the tagged version of $\mathrm{H} 3.3$ recapitulates the distribution pattern of the endogenous H3.3. Namely, we first observe a clear enrichment of $\mathrm{H} 3.3-\mathrm{HA}$ in the male pro- nucleus at early pronuclear stages whereas $\mathrm{H} 3.3-\mathrm{HA}$ is undetectable in the female pronucleus at the same stages. We first detect H3.3-HA in the female pronucleus at the PN3-PN4 stages, but the accumulation of $\mathrm{H} 3.3-\mathrm{HA}$ is still higher in the male pronucleus (Fig. 3A-C). At the PN5 stage the distribution of H3.3-HA is equivalent in the two pronuclei (Fig. 3A). Thus, these experiments confirm our observations using the H3.3 specific antibody. These 
A

PN0-PN1

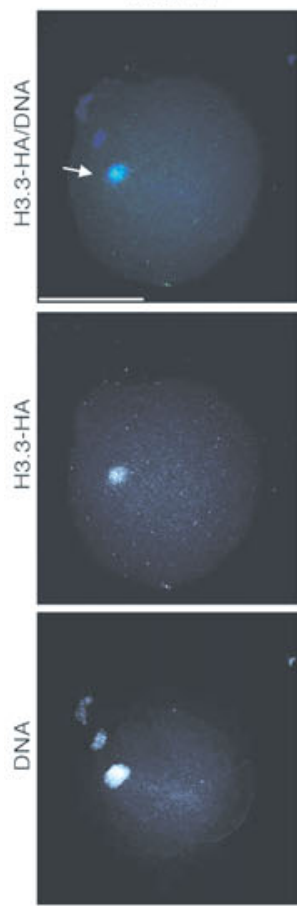

B

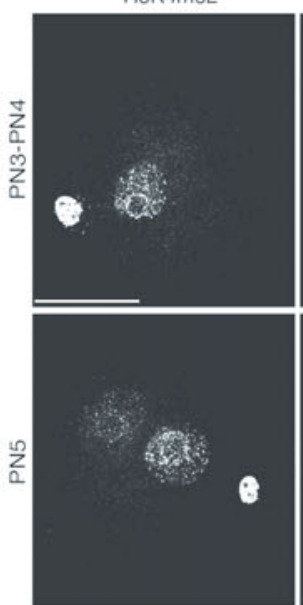

C
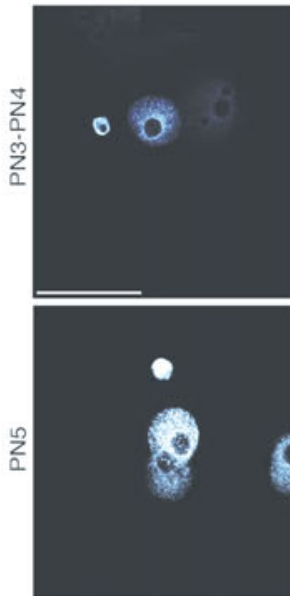

PN2

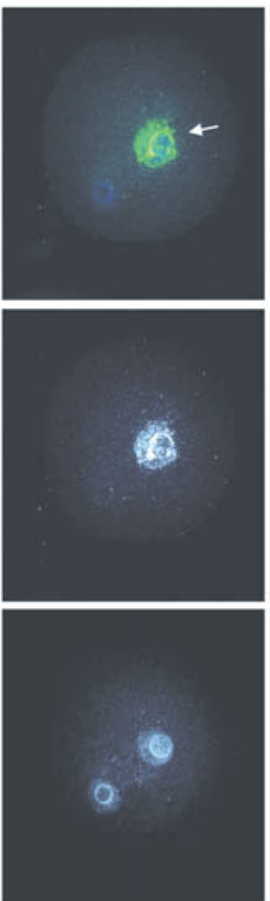

H3.3-HA

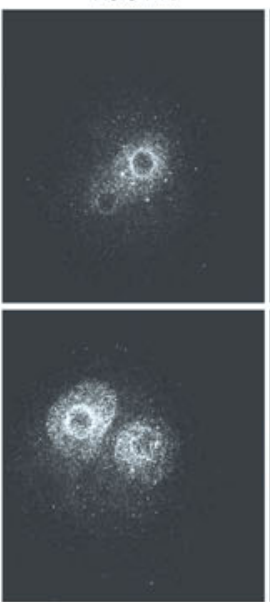

H3.3-HA

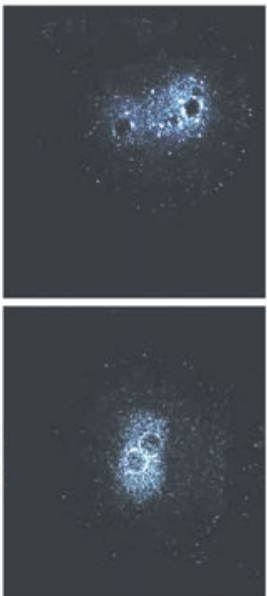

PN3-PN4
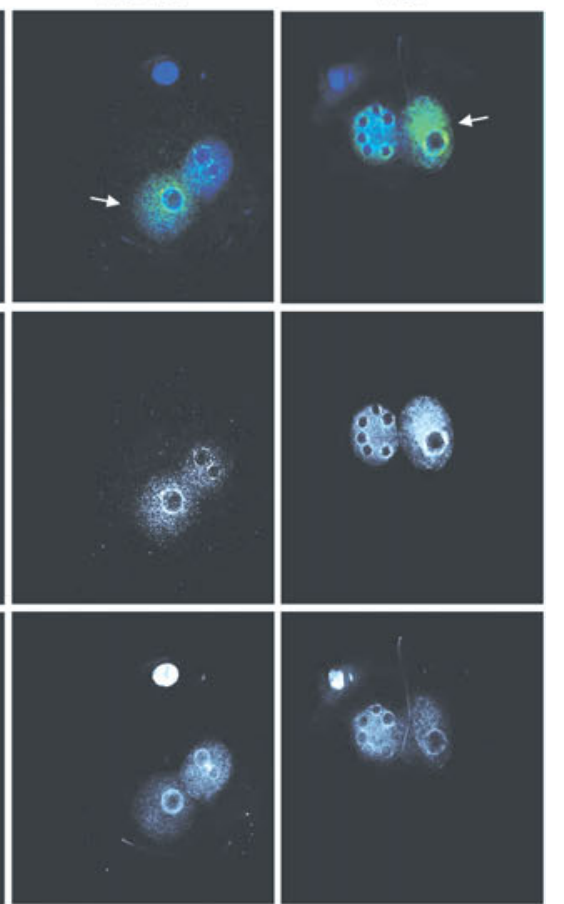

DNA

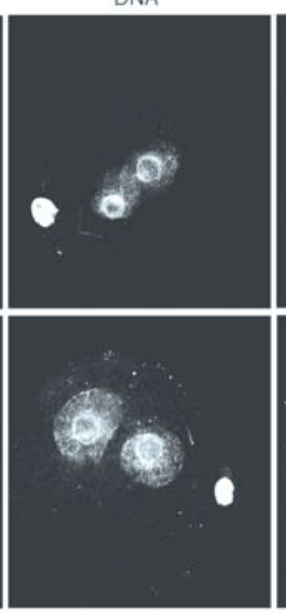

DNA

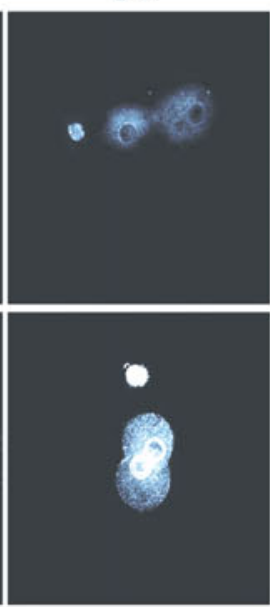

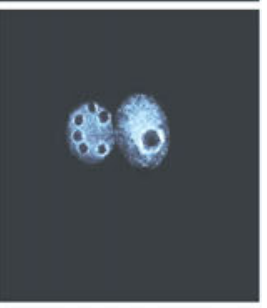

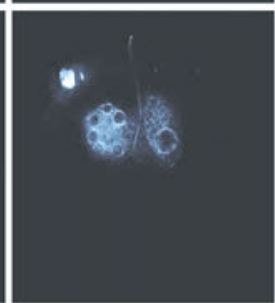

Merge
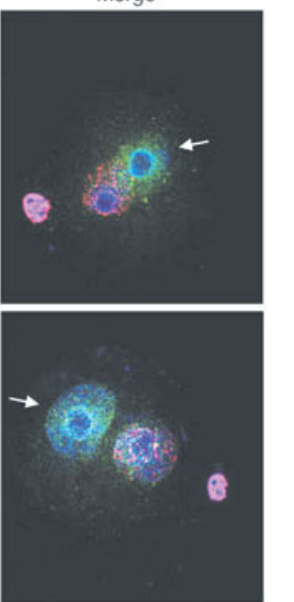

Merge

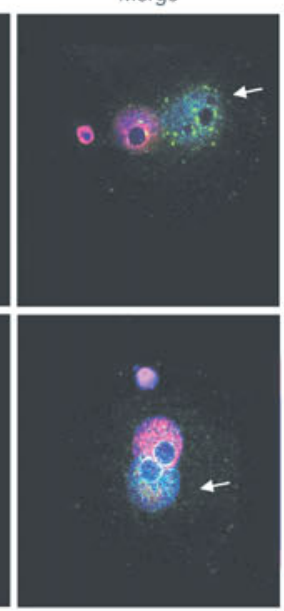

results also suggest that the preferential incorporation in the male pronucleus is inherent to H3.3, regardless of whether it is the endogenous protein or a tagged injected one. This indicates that there is no obvious requirement for pre-existing covalent modified $\mathrm{H} 3.3$ for its appropriate deposition.

The female pronucleus is enriched in mono and trimethylated $\mathrm{H} 3 \mathrm{~K} 4$, defining an epigenetic asymmetry between the two pronuclei, which disappears at the PN5 stage (Lepikhov and Walter, 2004)(Fig. $3 \mathrm{C})$. To unequivocally distinguish between the male and female pronucleus, we performed double immunostaining for $\mathrm{H} 3.3-\mathrm{HA}$ and $\mathrm{H} 3 \mathrm{~K} 4 \mathrm{me} 3$ from PN3 through PN5 stages. These experiments confirmed that the male pronucleus is enriched in H3.3HA (Fig. 3C). We also analysed the distribution of H3K4me2 from PN3 through PN5. We find that the levels of H3K4me2 are much higher in the female pronucleus, but in contrast to $\mathrm{H} 3 \mathrm{~K} 4 \mathrm{me} 3$, they remain asymmetric throughout all the pronuclear stages analysed (Fig. 3B). Unexpectedly thus, the female pronucleus is enriched in H3K4 mono, di and trimethylation, which are 'active' marks, whilst H3.3 is enriched in the male pronucleus. Moreover, we find that at the PN2 stage, when H3.3 is clearly restricted to the male pronucleus, $\mathrm{H} 3 \mathrm{~K} 4 \mathrm{me} 2$ is not detected in the paternal chromatin and the levels of $\mathrm{H} 3 \mathrm{~K} 4 \mathrm{me} 3$ are low (Fig. 4). This data suggests that in the mouse embryo, $\mathrm{H} 3.3$ is hypomethylated at $\mathrm{K} 4$ and that H3.3 may progressively become methylated as the first cell cycle progresses.

We then analysed the expression of histone H3.3 in 4-cell stage embryos and in the blastocyst. The histone H3.3 is readily detected in the four nuclei of the 4-cell stage embryos and the fluorescent signal is much stronger than in 2-cell stage embryos (Fig. 5A). H3.3 exhibits a widespread nuclear distribution with some accumulation in a few dots in the nucleoplasm and is absent from the densely DNA-stained heterochromatic regions and from around the nucleolar-like bodies (Fig. 5B). Thus, its localization is predominantly euchromatic at the 4-cell stage. In the blastocyst, H3.3 is also

Fig. 3. HA tagged H3.3 is incorporated first in the male pronucleus and the female pronucleus reaches equivalent levels only at the PN5 stage. (A) Distribution of HA-tagged H3.3 in the mouse zygote at different pronuclear stages. Zygotes were microinjected at the fertilisation cone stage with HA. H3.3 mRNA, cultured to the indicated pronuclear stages, fixed and processed for immunofluorescence using an anti HA antibody (green). $(\mathbf{B}, \mathbf{C})$ Embryos were processed as in $(A)$ but they were also stained with an antibody specific for dimethyl-(B) or trimethyl (C) H3K4 (red in the merge panel). The female pronucleus is distinguishable due to the enrichment in methylated H3K4. In all panels DNA is shown in blue in the merge composites (top). Shown are stack Z-series projections of representative embryos. Scale bar represents $50 \mu \mathrm{m}$. The male pronucleus is indicated with an arrow and the pronuclear stages are indicated. 
widely expressed in the nuclei of all cells. We do not detect any obvious difference in the $\mathrm{H} 3.3$ content between the inner cell mass and the trophectoderm (Fig. 5A). Analysis of blastocyst cells under higher magnification revealed that $\mathrm{H} 3.3$ was widespread in the nucleoplasm but accumulated within two foci of much greater intensity in each nucleus of both the inner cell mass (not shown) and the trophectoderm (Fig. 5C). Thus, in the blastocyst, the pattern of nuclear distribution of histone H3.3 is also different compared to the one in the 4-cell stage embryo.

\section{Discussion}

The mouse embryo undergoes reprogramming of its epigenetic marks during the first cell cycles (Li, 2002, Morgan etal., 2005). The epigenetic status of each cell within the embryo will be determined not only by differences in DNA methylation and histone modifications, but also by the content of histone and histone variants. Here we have shown that the histone $\mathrm{H} 3$ variant, $\mathrm{H} 3.3$, is deposited specifically in the male pronucleus. Moreover, there seems to be a dicotomy since the male pronucleus does not have active methylation marks in $\mathrm{K} 4$ of histone $\mathrm{H} 3$ at the early stages of pronuclear formation.

The zygote gradually acquires a permissive state for transcription (Latham et al., 1992). Thus, the alterations in chromatin structure that follow fertilisation could contribute to rendering the zygote transcriptionally competent. The observed incorporation of H3.3, which has been reported to be enriched at active genes and to contain active marks (McKittrick et al., 2004, Schwartz and Ahmad, 2005), could contribute to the acquisition of this state.

Asymmetries in the epigenetic status of the parental genomes occur in the zygote. These include active DNA demethylation in the paternal genome but not in the maternal one and accumulation of H3K4me1, H3K9me2 and H3K27me3 in the female pronucleus (Arney et al., 2002, Lepikhov and Walter, 2004, Santos et al., 2005). In contrast, the male pronucleus displays higher levels of histone $\mathrm{H} 4$ acetylation than the maternal chromatin (Adenot et al., 1997). However, histone $\mathrm{H} 4$ acetylation and methylation of H3K4 reach similar levels in both pronuclei at the end of the zygote stage and the main differences between them remain in heterochromatic structures and imprinting (Santos et al., 2005). Here we show, consistent with the differences in histone modifications, that there is also an asymmetry in the incorporation of H3.3 into the pronuclei. This asymmetry disappears at the late zygote stage. Our results are in line with recent work by van der Heijden and colleagues, who reported enrichment of $\mathrm{H} 3.1$ in the female pronucleus but not in the male one, leading the authors to suggest that the male pronucleus would be enriched in H3.3 (van der Heijden et al., 2005). Changes in the histone composition in the male pronucleus may be necessary for the first wave of transcriptional activation that starts first in the paternal genome, which supports higher levels of transcription than the maternal pronucleus (Aoki et al., 1997, Bouniol et al., 1995, Bouniol-Baly et al., 1997). Additionally, the differential incorporation of $\mathrm{H} 3.3$ into the pronuclei may also contribute to 'delay' the incorporation of repressive marks into the paternal genome (Arney et al., 2002, Lepikhov and Walter, 2004, Santos et al., 2005).

We observed changes in the pattern of nuclear distribution of histone H3.3. A characteristic punctate pattern found at the 2-cell stage changes into a more widely dispersed at the 4-cell stage. In the blastocyst, we observed a characteristic enrichment in two

spots in the nucleoplasm. It is interesting to note that these changes correlate with the three major changes in transcript profile in the embryo: the first one up to the 2-cell stage, the second one after the 4-cell stage and the third one that leads to cellular differentiation in the blastocyst (Hamatani et al., 2004, Wang et al., 2004).

Deposition of histone H3.3 in somatic cells can occur in both replication-dependent or independent mechanisms (Ahmad and Henikoff, 2002a, Ahmad and Henikoff, 2002b). Histones are first detected in the male pronucleus at the early PN1 stage (Adenot et al., 1997, Arney et al., 2002, Lepikhov and Walter, 2004). We observed incorporation of $\mathrm{H} 3.3$ in the male pronucleus around the PN2 stage, before the onset of transcription activation, suggesting that the deposition of H3.3 in the zygote may not (at least initially) be coupled to genome activation. Indeed, HIRA, which is known to mediate H3.3 deposition in somatic cells (Tagami et al., 2004) was recently reported to be present in the sperm nucleus during decondensation immediately after fertilization and before genome activation (van der Heijden et al., 2005). Given that replication in the zygote starts at $\sim 21 \mathrm{~h}$ phCG and that BrdU incorporation is not detected in PN1 and PN2 embryos (Adenot et al., 1997, BouniolBaly et al., 1997), it is possible that H3.3 incorporation is also independent of DNA synthesis. Morever, the punctate localization pattern that we observed for H3.3 in the zygote persists until the late 2-cell stage when two rounds of replication have already occurred. So it is conceivable that the incorporation of H3.3 in the zygote occurs by a different mechanism independent of replication and transcription. A recent report has indeed documented that in Drosophila, HIRA is required for removal of the protamines (Jayaramaiah Raja and Renkawitz-Pohl, 2005) and for chromatin assembly in the male pronucleus (Loppin et al., 2005). Moreover, maternal H3.3-Flag specifically accumulates in the male pronucleus, suggestive of a replication independent nucleosome assembly at the genome wide level.

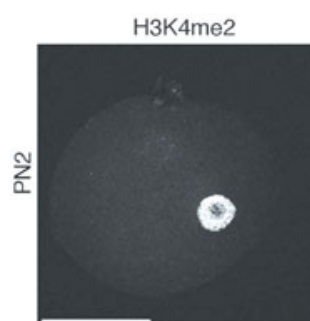

$\mathrm{H} 3 \mathrm{~K} 4 \mathrm{me} 3$

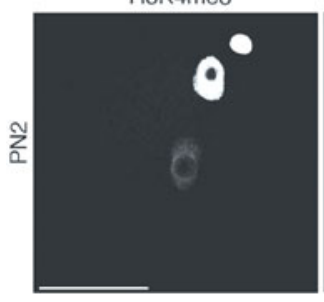

Fig. 4. The distribution of di- and trimethylated $\mathrm{H} 3 \mathrm{~K} 4$ at the earlier pronuclear stages is assymetric. Zygotes were collected at the PN2 stage, fixed and stained with specific methyl antibodies as indicated (red). DNA is shown in blue. Shown are stack Z-series projections of representative embryos. The male pronucleus is depicted by an arrow. Scale bar represents $50 \mu \mathrm{m}$. Note that the levels of $\mathrm{H} 3 \mathrm{~K} 4 \mathrm{me} 3$ in the male pronucleus are low but detectable, whereas those of $\mathrm{H} 3 \mathrm{~K} 4 \mathrm{me} 2$ are undetectable. 
A
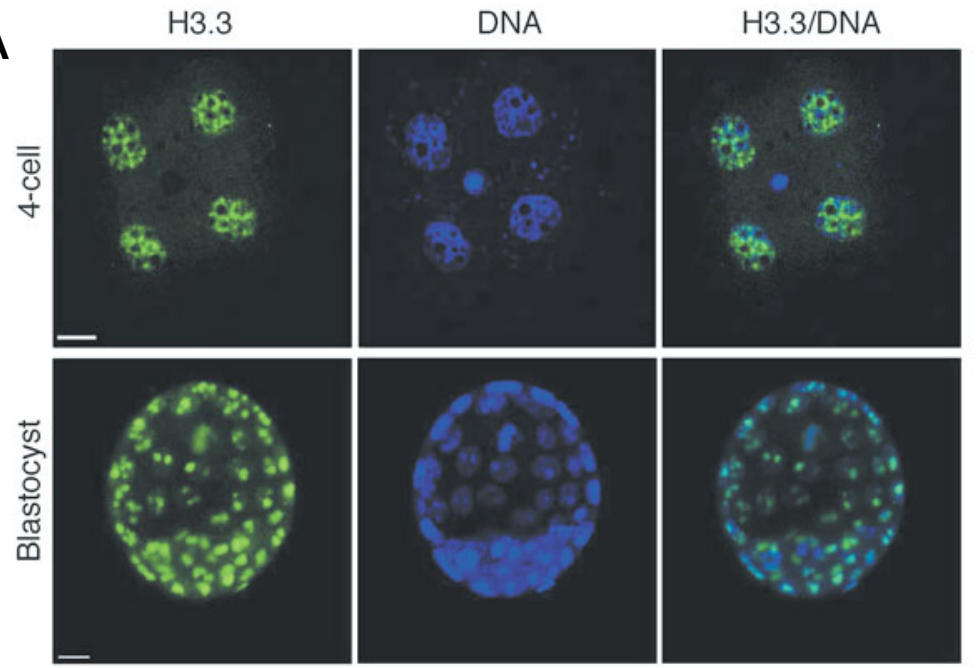
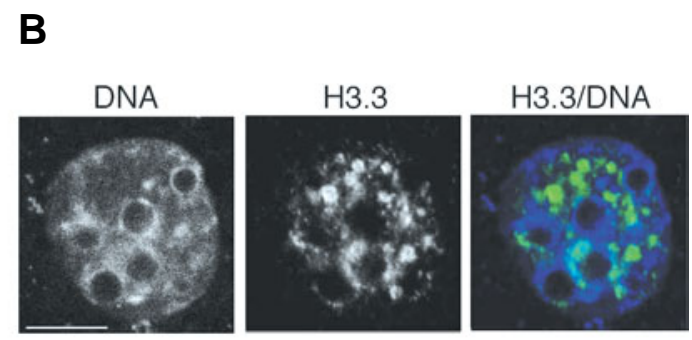

C
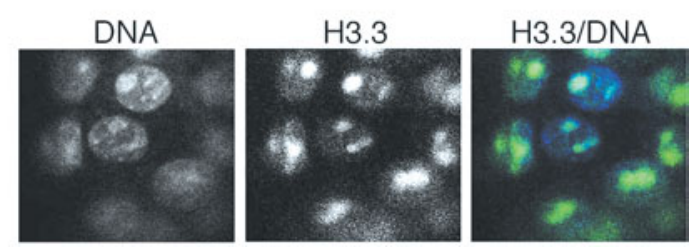

Fig. 5. Distribution of H3.3 in mouse 4-cell stage embryos and blastocyst. (A) Embryos were collected from the oviduct, processed for immunofluorescence with the H3.3 antibody and analysed under confocal microscopy. H3.3 is shown in green, DNA in blue. Shown are stack, single channel and merge confocal sections of representative embryos at the indicated stages. Scale bar represents $10 \mu \mathrm{m}$. (B) Higher magnification of one of the nuclei of the 4-cell stage embryo shown in (A). Note that H3.3 is distributed throughout the nucleoplasm but is absent from the densely stained heterochromatic regions. Shown are single confocal sections from single channel acquisitions and the corresponding merge image. Scale bar represents $10 \mu \mathrm{m}$. (C) Trophectoderm cells of the blastocyst shown in (A). Representative sections derived from single channel acquisition and merge image are shown.

Genetic ablation of some of the histone modifiers that are involved in the establishment of epigenetic asymmetries in the zygote such as Enhancer of Zeste 2 results in impaired development (Erhardt et al., 2003, Santos et al., 2002). A hypomorphic mutation of the H3f3a gene, coding for H3.3, results in male subfertility (Couldrey et al., 1999). Thus, it will be interesting to determine whether $\mathrm{H} 3.3$ has a role in the reprogramming of the parental genomes that follow fertilisation. Investigating not only the changes in chromatin modifications but also histone composition during this period, will help to understand the mechanisms underlying these events. Our results, which analyse the distribution of histone H3.3 throughout preimplantation development make a contribution towards this goal.

\section{Experimental Procedures}

\section{Embryo collection and culture}

Embryos were collected from F1 (C57BL/6 $\times$ CBA/H) $\sim 6$ weeks old superovulated females that were crossed with $F 1$ males as described (Hogan et al., 1994). Zygotes and cleavage stage embryos were collected at the indicated hours post-hCG ( $\mathrm{h}$ phCG). Oocytes were recovered from $\sim 6$ weeks old $\mathrm{F} 1$ females as described by Hogan et al (Hogan et al., 1994). Experiments with animals were carried out according to the Home Office regulation. Pronuclear stages (PN) were classified according to Adenot et al (1997; Tablel and Fig. 3 therein, according to both, post-hCG time and the distance between pronuclei) and corresponded approximately to the following times after hCG injection in our strain and Animal Facility: PNOPN1, 18h phCG; PN2, 20h phCG; PN3-PN4, 24h phCG; PN5, 27h phCG. For the BrUTP experiments, we refer to the hCG timing throughout the text so that the experimental design is clearer to understand.

\section{Immunostaining and confocal analysis}

After removal of the zona pellucida with acid Tyrode's solution (Sigma), embryos were washed three times in PBS and fixed in 5\% paraformaldehyde, $0.04 \%$ Triton, $0.3 \%$ Tween and $0.2 \%$ sucrose in PBS for 20 minutes at $37^{\circ} \mathrm{C}$. After permeabilisation with $0.5 \%$ Triton in PBS for 20 minutes, the embryos were washed three times in PBS-T ( $0.1 \%$ Tween in PBS), blocked in 3\% BSA in PBS-T and incubated with the primary antibodies (H3.3 ab4263, abcam, 1:75 dilution; anti histone $\mathrm{H} 3$ dimethyl $\mathrm{K} 4 \mathrm{ab} 7766$, abcam, 1:150 dilution; anti histone $\mathrm{H} 3$ trimethyl $\mathrm{K} 4$ ab8580, 1:150 dilution; anti HA antibody clone $3 \mathrm{~F} 10$, Roche, $1: 500$ dilution) for $\sim 12 \mathrm{~h}$ at $4^{\circ} \mathrm{C}$. It is essential to note that we used an early batch of the $\mathrm{H} 3.3$ antibody, which was affinity purified from the first serum. This batch was fully characterized (Fig. 1A) and was shown to recognise nucleosomal histone $\mathrm{H} 3.3$ in vivo in ChIP assays by two independent laboratories (Johnson et al., 2004; Daujat, S. personnal communication). Embryos were then washed twice in PBS-T, blocked for 30 minutes and incubated for $2 \mathrm{~h}$ at $25^{\circ} \mathrm{C}$ with the corresponding secondary antibodies (FITC-conjugated donkey anti rabbit IgG, Jackson ImmunoResearch). After 2 washes in PBS-T, the DNA was stained with TOTO-3 (Molecular Probes) and the embryos were mounted in Vectashield (Vector Laboratories). Confocal microscopy was performed using a $40 x$ oil objective in a BioRad 1024 inverted microscope (Blastocysts) or a 60x oil objective in a BioRad Radiance Upright Confocal Laser Microscope using the BioRad LaserSharp 2000 software (all the other stages). All the stainings were repeated independently at least two times with at least 10 embryos analysed per stage.

\section{BrUTP labelling}

BrUTP labelling was performed as described (Borsuk and Maleszewski, 2002)(Torres-Padilla and Zernicka-Goetz, submitted). Embryos were collected at 20h phCG and microinjected using an Eppendorf Transjector 5246 with 1-2 pl of $100 \mathrm{mM} \mathrm{BrUTP}$ (5-bromo UTP, Sigma) in 2mM PIPES, $140 \mathrm{mM} \mathrm{KCl}, \mathrm{pH} 7.4$. Embryos were fixed after culture at the time equivalent to 24 and $27 \mathrm{~h} \mathrm{phCG}$ and processed for immunostaining using an anti-BrdU antibody (1:100 dilution, Sigma).

\section{mRNA microinjection}

Zygotes were collected at the fertilisation cone stage ( 17h phCG) and microinjected with $1-2 \mathrm{pl}$ of $500 \mathrm{ng} / \mu \mathrm{l}$ of C-terminal double HA-tagged (human) histone H3.3 mRNA capped and transcribed in vitro. Zygotes were then cultured in $\mathrm{KSOM}$ under a $\% 5 \mathrm{CO}_{2}$ atmosphere at $37^{\circ} \mathrm{C}$, fixed at the different pronuclear stages as indicated in the figure legends and 
processed for immunofluorescence. At least 20 embryos per stage from two independent experiments were analysed.

\section{Western blot}

Two micrograms of core histone preparation (500 ng of each, SIGMA) or $1 \mu \mathrm{g}$ of purified bacterially produced histone $\mathrm{H} 3.2$ were resolved by SDS-PAGE, blotted to nitrocellulose and probed O.N. with the anti H3.3 antibody (1:1000 dilution). Competitor peptides were added as indicated at a concentration of $1 \mu \mathrm{g} / \mathrm{ml}$. The H3.3 antibody used (ab4263, Batch 34119) was obtained from Abcam Ltd., UK. It was thoroughly characterised with regards to its specificity. Due to production issues, it is no longer commercially available.

\section{Acknowledgments}

This work was supported by the Wellcome Trust. M.Z-G. is a Wellcome Senior Research Fellow. M-E.-T-P. is a Long Term EMBO fellow.

\section{References}

ADENOT, P.G., MERCIER, Y., RENARD, J.P. and THOMPSON, E.M. (1997). Differential $\mathrm{h} 4$ acetylation of paternal and maternal chromatin precedes DNA replication and differential transcriptional activity in pronuclei of 1-cell mouse embryos. Development 124: 4615-25.

AHMAD, K. and HENIKOFF, S. (2002a). Histone h3 variants specify modes of chromatin assembly. Proc Natl Acad Sci USA 99 Suppl 4: 16477-84.

AHMAD, K. and HENIKOFF, S. (2002b). The histone variant h3.3 marks active chromatin by replication-independent nucleosome assembly. Mo/ Cel/9: 1191200.

AOKI, F., WORRAD, D.M. and SCHULTZ, R.M. (1997). Regulation of transcriptional activity during the first and second cell cycles in the preimplantation mouse embryo. Dev Bio/181: 296-307.

ARNEY, K.L., BAO, S., BANNISTER, A.J., KOUZARIDES, T. and SURANI, M.A. (2002). Histone methylation defines epigenetic asymmetry in the mouse zygote. Int J Dev Bio/46: 317-20.

BORSUK, E. and MALESZEWSKI, M. (2002). DNA replication and rna synthesis in thymocyte nuclei microinjected into the cytoplasm of artificially activated mouse eggs. Zygote 10: 229-38.

BOUNIOL, C., NGUYEN, E. and DEBEY, P. (1995). Endogenous transcription occurs at the 1-cell stage in the mouse embryo. Exp Cell Res 218: 57-62.

BOUNIOL-BALY, C., NGUYEN, E., BESOMBES, D. and DEBEY, P. (1997). Dynamic organization of DNA replication in one-cell mouse embryos: Relationship to transcriptional activation. Exp Cell Res 236: 201-11.

COULDREY, C., CARLTON, M.B., NOLAN, P.M., COLLEDGE, W.H. and EVANS, M.J. (1999). A retroviral gene trap insertion into the histone 3.3a gene causes partial neonatal lethality, stunted growth, neuromuscular deficits and male subfertility in transgenic mice. Hum Mol Genet 8: 2489-95.

ERHARDT, S., SU, I.H., SCHNEIDER, R., BARTON, S., BANNISTER, A.J., PEREZ-BURGOS, L., JENUWEIN, T., KOUZARIDES, T., TARAKHOVSKY, A. and SURANI, M.A. (2003). Consequences of the depletion of zygotic and embryonic enhancer of zeste 2 during preimplantation mouse development. Development 130: 4235-48.

GAUTIER, T., ABBOTT, D.W., MOLLA, A., VERDEL, A., AUSIO, J. and DIMITROV, S. (2004). Histone variant h2abbd confers lower stability to the nucleosome. EMBO Rep 5: 715-20.

HAMATANI, T., CARTER, M.G., SHAROV, A.A. and KO, M.S. (2004). Dynamics of global gene expression changes during mouse preimplantation development. Dev Cel/6: 117-31.
HOGAN, B.L., BEDDINGTON, R., COSTANTINI, F. and LACY, E. (1994). Manipulating the mouse embryo. Cold Spring Harbor Laboratory Press.

JANICKI, S.M., TSUKAMOTO, T., SALGHETTI, S.E., TANSEY, W.P., SACHIDANANDAM, R., PRASANTH, K.V., RIED, T., SHAV-TAL, Y., BERTRAND, E., SINGER, R.H. et al. (2004). From silencing to gene expression: Real-time analysis in single cells. Cel/116: 683-698.

JAYARAMAIAH RAJA, S. and RENKAWITZ-POHL, R. (2005). Replacement by drosophila melanogaster protamines and mst $77 \mathrm{f}$ of histones during chromatin condensation in late spermatids and role of sesame in the removal of these proteins from the male pronucleus. Mol Cell Bio/25: 6165-77.

KAMAKAKA, R.T. and BIGGINS, S. (2005). Histone variants: Deviants? Genes Dev 19: 295-310.

LATHAM, K.E., SOLTER, D. and SCHULTZ, R.M. (1992). Acquisition of a transcriptionally permissive state during the 1-cell stage of mouse embryogenesis. Dev Biol 149: 457-62.

LEPIKHOV, K. and WALTER, J. (2004). Differential dynamics of histone h3 methylation at positions k4 and k9 in the mouse zygote. BMC Dev Bio/4: 12.

$\mathrm{LI}$, E. (2002). Chromatin modification and epigenetic reprogramming in mammalian development. Nat Rev Genet 3: 662-73.

LOPPIN, B., BONNEFOY, E., ANSELME, C., LAURENCON, A., KARR, T.L. and COUBLE, P. (2005). The histone h3.3 chaperone hira is essential for chromatin assembly in the male pronucleus. Nature 437: 1386-90.

MCKITTRICK, E., GAFKEN, P.R., AHMAD, K. and HENIKOFF, S. (2004). Histone h3.3 is enriched in covalent modifications associated with active chromatin. Proc Natl Acad Sci USA 101: 1525-30.

MORGAN, H.D., SANTOS, F., GREEN, K., DEAN, W. and REIK, W. (2005). Epigenetic reprogramming in mammals. Hum Mol Genet $14 \mathrm{Spec}$ No 1: R47-58.

SANTOS, F., HENDRICH, B., REIK, W. and DEAN, W. (2002). Dynamic reprogramming of DNA methylation in the early mouse embryo. Dev Bio/241: 172-82.

SANTOS, F., PETERS, A.H., OTTE, A.P., REIK, W. and DEAN, W. (2005). Dynamic chromatin modifications characterise the first cell cycle in mouse embryos. Dev Bio/280: 225-36.

SARMENTO, O.F., DIGILIO, L.C., WANG, Y., PERLIN, J., HERR, J.C., ALLIS, C.D. and COONROD, S.A. (2004). Dynamic alterations of specific histone modifications during early murine development. J Cell Sci117: 4449-59.

SCHWARTZ, B.E. and AHMAD, K. (2005). Transcriptional activation triggers deposition and removal of the histone variant h3.3. Genes Dev 19: 804-14.

TAGAMI, H., RAY-GALLET, D., ALMOUZNI, G. and NAKATANI, Y. (2004). Histone $\mathrm{H} 3.1$ and $\mathrm{H} 3.3$ complexes mediate nucleosome assembly pathways dependent or independent of DNA synthesis. Cel/116: 51-61.

VAN DER HEIJDEN, G.W., DIEKER, J.W., DERIJCK, A.A., MULLER, S., BERDEN, J.H., BRAAT, D.D., VAN DER VLAG, J. and DE BOER, P. (2005). Asymmetry in histone $h 3$ variants and lysine methylation between paternal and maternal chromatin of the early mouse zygote. Mech Dev 122: 1008-22.

WANG, Q.T., PIOTROWSKA, K., CIEMERYCH, M.A., MILENKOVIC, L., SCOTT, M.P., DAVIS, R.W. and ZERNICKA-GOETZ, M. (2004). A genome-wide study of gene activity reveals developmental signaling pathways in the preimplantation mouse embryo. Dev Cel/6: 133-44.

WIEKOWSKI, M., MIRANDA, M., NOTHIAS, J.Y. and DEPAMPHILIS, M.L. (1997). Changes in histone synthesis and modification at the beginning of mouse development correlate with the establishment of chromatin mediated repression of transcription. J Cel/ Sci 110 (Pt 10): 1147-58.

Received: July 2005

Reviewed by Referees: September 2005

Modified by Authors and Accepted for Publication: January 2006 